\title{
Novel Multiband Linearization Technique for Closely-Spaced Dual-Band Signals of Wide Bandwidth
}

\author{
Ying Liu*\#, Patrick Roblin*, Hai Yu*, Shihai Shao\#, and Youxi Tang\# \\ *Department of Electrical \& Computer Engineering, OSU, Columbus, 43210, USA \\ \# National Key Lab of Science \& Technology on Communications, UESTC, Chengdu, 611731, China
}

\begin{abstract}
In the concurrent dual-band digital predistortion (DPD), it is required that each band of interest be accurately measured, so that the in-band distortion can be modeled and suppressed. However when the two bands are closely spaced, the inter-band modulation by-products overlap with the crossmodulation and the in-band intermodulation, thus leading to linearization performance degradation. On the other hand, by directly applying the single band linearization for closely-spaced dual-band signals, the one-band DPD approach will saturate in performance due to the wide bandwidth as well as the high nonlinear order and memory depth required resulting in an ill-conditioning problem. To resolve the spectral overlaps taking place in closely-spaced dual wide-band system, (1) the in-band intermodulation, (2) the cross-modulation, and (3) the inter-band modulation by-products are jointly extracted in the new proposed dual-band PA characterization scheme to yield an improved frequency/time selective modeling. The signals of interest extracted in each band using the proposed model are then used for dual-band DPD coefficient extraction. Improved modeling and linearization performances by up to $5 \mathrm{~dB}$ are verified with experiments performed on two 40-MHz LTEadvanced signals $40 \mathrm{MHz}$ apart.
\end{abstract}

Index Terms-Digital predistortion, concurrent dual-band, memory polynomial, power amplifier.

\section{INTRODUCTION}

Modern wireless communication systems are continuously developing in data rate and bandwidth to support more users and provide more data services, e.g., the Long Term Evolution (LTE)-advanced system are employing orthogonal frequency division multiplexing (OFDM) to achieve high peak data rate of $1 \mathrm{~Gb} / \mathrm{s}$ in the downlink with a maximum instantaneous bandwidth of $100 \mathrm{MHz}$ [1]. To further fully utilize the frequency spectrum and support the significant increase of the modulated signal bandwidth, concurrent dual-band amplifiers have been developed recently [2].

As the bandwidth increases, it poses stringent requirements on the power amplifier (PA) linearity and efficiency. Digital predistortion (DPD) has proven to be one of the most costeffective methods to compensate for the PA nonlinearity and memory effect in order to suppress the spectral regrowth [3]. However, in concurrent dual-band transmitters, directly applying the conventional one-band DPD technique on the full band results in large transmitting bandwidth which requires analogto-digital converters (ADCs) and digital-to-analog converters (DACs) with prohibitively high sampling rates.

Recently, several dual-band linearization methods have been proposed to compensate for the PA nonlinearities and memory effect in dual-band cases [4]-[7]. The authors in [4] proposed a frequency-selective linearization technique based on
PA characteristics measurement using a large-signal network analyzer (LSNA). It successfully removes in-band distortion and inter-modulation distortion up to the third order. Bassam et al. [5] proposed a 2D digital predistortion (2D-DPD) architecture, in which the radio frequency (RF) signals in each band are captured and digitized separately. The authors also proposed a multi-cell processing digital predistortion (MCPDPD) architecture [7], where the nonlinearities and memory effects of multi-carrier signals in each frequency band are compensated for independently of the signals in the other frequency bands. All these methods can significantly reduce the bandwidth and sampling rate requirement because the signal bands are widely located, thus the bands of interest and the corresponding distortions can be captured separately by means of filtering.

However, when the transmitting signal bands are closely spaced, the in-band intermodulation, the cross-modulation, and the inter-band modulation by-products overlap with each other. In this case, the band of interest and its distortion cannot be simply isolated using filtering. This introduces an error which corrupts the DPD model extraction process and degrades the linearization performances.

In this paper, we propose a novel multiband linearization technique to handle the dual-band signals with closely-spaced bands. A novel multiband PA model based on dual-band memory polynomials is proposed, which jointly extracts the in-band intermodulation, the cross-modulation, and the interband modulation by-products. Using this new joint model estimation for the two bands of interest instead of separating them by filtering, the DPD coefficients for the adjacent bands can be extracted by using the indirect learning architecture. Experiments are performed on two 40-MHz LTE-advanced signals spaced by $40 \mathrm{MHz}$ to demonstrate the effectiveness of this new method.

\section{Multiband Linearization FOR DUAL-BAND Signal}

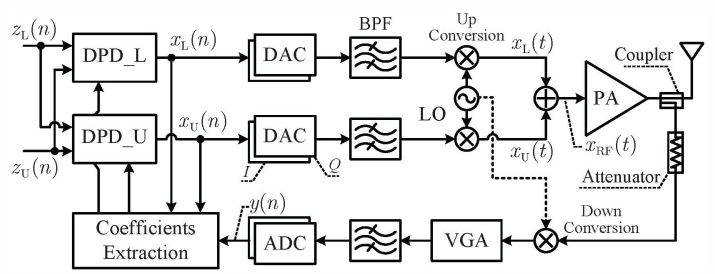

Fig. 1. Block diagram of the proposed multiband DPD system. 
The proposed block diagram of the proposed multiband DPD system is illustrated in Fig. 1. The dual-band source signal is composed of two signals $x_{\mathrm{L}}(n)$ and $x_{\mathrm{U}}(n)$. The composite signal $x_{\mathrm{RF}}(t)$ can be expressed as

$$
x_{\mathrm{RF}}(t)=\mathfrak{R}\left\{x_{\mathrm{L}}(t) e^{j w_{\mathrm{L}} t}+x_{\mathrm{U}}(t) e^{j w_{\mathrm{U}} t}\right\},
$$

where $\mathfrak{R}\{\cdot\}$ is the real part of a signal, $w_{\mathrm{L}}$ and $w_{\mathrm{U}}$ represent the frequency centers of the lower and upper band, respectively. The output of the PA around the fundamental band, without considering the output harmonics, can be obtained as [8]

$$
\begin{aligned}
& y_{\mathrm{RF}}(t)=\Re\left\{y_{\mathrm{L} 1} e^{j w_{\mathrm{L}} t}+y_{\mathrm{L} 3} e^{j\left(2 w_{\mathrm{L}}-w_{\mathrm{U}}\right) t}+y_{\mathrm{L} 5} e^{j\left(3 w_{\mathrm{L}}-2 w_{\mathrm{U}}\right) t}\right. \\
& \left.+y_{\mathrm{U} 1} e^{j w_{\mathrm{U}} t}+y_{\mathrm{U} 3} e^{j\left(2 w_{\mathrm{U}}-w_{\mathrm{L}}\right) t}+y_{\mathrm{U} 5} e^{j\left(3 w_{\mathrm{U}}-2 w_{\mathrm{L}}\right) t}\right\}
\end{aligned}
$$

where a nonlinear order of five is considered. In the baseband, the in-band intermodulation $y_{\mathrm{L} 1}$ and $y_{\mathrm{U} 1}$ and the inter-band modulation by-products $y_{\mathrm{L} 3}, y_{\mathrm{L} 5}, y_{\mathrm{U} 3}$, and $y_{\mathrm{U} 5}$ are given by

$$
\begin{aligned}
& y_{\mathrm{L} 1}=x_{\mathrm{L}}(n) \times f_{\mathrm{L} 1}\left(\left|x_{\mathrm{L}}(n)\right|^{2},\left|x_{\mathrm{U}}(n)\right|^{2}\right) \\
& y_{\mathrm{U} 1}=x_{\mathrm{U}}(n) \times f_{\mathrm{U} 1}\left(\left|x_{\mathrm{L}}(n)\right|^{2},\left|x_{\mathrm{U}}(n)\right|^{2}\right) \\
& y_{\mathrm{L} 3}=x_{\mathrm{L}}^{2}(n) x_{\mathrm{U}}^{*}(n) \times f_{\mathrm{L} 3}\left(\left|x_{\mathrm{L}}(n)\right|^{2},\left|x_{\mathrm{U}}(n)\right|^{2}\right) \\
& y_{\mathrm{U} 3}=x_{\mathrm{U}}^{2}(n) x_{\mathrm{L}}^{*}(n) \times f_{\mathrm{U} 3}\left(\left|x_{\mathrm{L}}(n)\right|^{2},\left|x_{\mathrm{U}}(n)\right|^{2}\right) \\
& y_{\mathrm{L} 5}=x_{\mathrm{L}}^{3}(n) x_{\mathrm{U}}^{* 2}(n) \times f_{\mathrm{L} 5}\left(\left|x_{\mathrm{L}}(n)\right|^{2},\left|x_{\mathrm{U}}(n)\right|^{2}\right) \\
& y_{\mathrm{U} 5}=x_{\mathrm{U}}^{3}(n) x_{\mathrm{L}}^{* 2}(n) \times f_{\mathrm{U} 5}\left(\left|x_{\mathrm{L}}(n)\right|^{2},\left|x_{\mathrm{U}}(n)\right|^{2}\right),
\end{aligned}
$$

where $f_{\mathrm{Lm}}\left(\left|x_{\mathrm{L}}(n)\right|^{2},\left|x_{\mathrm{U}}(n)\right|^{2}\right)$ and $f_{\mathrm{Um}}\left(\left|x_{\mathrm{L}}(n)\right|^{2},\left|x_{\mathrm{U}}(n)\right|^{2}\right)$ are the nonlinear gain functions at different order of interband modulation. It should be noted that memory effects are not illustrated here, but can be simply included in this model by replacing $n$ with $(n-q), n=0,1, \cdots, Q-1$. Currently, the conventional frequency-selective approach proposed in [5], [6], focus only on the in-band intermodulation and the crossmodulation between the two bands, i.e., only $y_{\mathrm{L} 1}(n)$ and $y_{\mathrm{U} 1}(n)$ terms are included in the their dual-band PA models, while filtering out the inter-band modulations. When the bands are closely separated, the in-band intermodulation, the crossmodulation, and the inter-band modulation by-products will overlap with each other, making the conventional dual-band DPD model extraction process unreliable.

In the proposed multiband PA model, we include all the inter-band modulation terms into the dual-band PA model. Thus, the PA model for the lower band can be expressed as

$$
\begin{aligned}
& y_{\mathrm{L}}(n)=y_{\mathrm{L} 1}(n)+y_{\mathrm{L} 3}(n) e^{-j w_{\Delta} n T_{s}}+y_{\mathrm{L} 5}(n) e^{-j 2 w_{\Delta} n T_{s}} \\
& =\sum_{m=0}^{Q-1} x_{\mathrm{L}}(n-q) f_{\mathrm{L} 1}\left(\left|x_{\mathrm{L}}(n-q)\right|^{2},\left|x_{\mathrm{U}}(n-q)\right|^{2}\right) \\
& +e^{-j w_{\Delta} n T_{s}} \sum_{m=0}^{Q-1} x_{\mathrm{L}}^{2}(n-q) x_{\mathrm{U}}^{*}(n-q) f_{\mathrm{L} 3}\left(\left|x_{\mathrm{L}}(n-q)\right|^{2},\left|x_{\mathrm{U}}(n-q)\right|^{2}\right) \\
& +e^{-j 2 w_{\Delta} n T_{s}} \sum_{m=0}^{Q-1} x_{\mathrm{L}}^{3}(n-q) x_{\mathrm{U}}^{* 2}(n-q) f_{\mathrm{L} 5}\left(\left|x_{\mathrm{L}}(n-q)\right|^{2},\left|x_{\mathrm{U}}(n-q)\right|^{2}\right),
\end{aligned}
$$

where $w_{\Delta}=w_{\mathrm{U}}-w_{\mathrm{L}}$ is the frequency separation between the centers of the upper and lower bands, $T_{s}$ is the sampling period. Similarly, the PA model for the upper band can be expressed as

$$
\begin{aligned}
& y_{\mathrm{U}}(n)=y_{\mathrm{U} 1}(n)+y_{\mathrm{U} 3}(n) e^{j w_{\Delta} n T_{s}}+y_{\mathrm{U} 5}(n) e^{j 2 w_{\Delta} n T_{s}} \\
& =\sum_{m=0}^{Q-1} x_{\mathrm{U}}(n-q) f_{\mathrm{U} 1}\left(\left|x_{\mathrm{U}}(n-q)\right|^{2},\left|x_{\mathrm{L}}(n-q)\right|^{2}\right) \\
& +e^{j w_{\Delta} n T_{s}} \sum_{q=0}^{Q-1} x_{\mathrm{U}}^{2}(n-q) x_{\mathrm{L}}^{*}(n-q) f_{\mathrm{U} 3}\left(\left|x_{\mathrm{U}}(n-q)\right|^{2},\left|x_{\mathrm{L}}(n-q)\right|^{2}\right) \\
& +e^{j 2 w_{\Delta} n T_{s}} \sum_{q=0}^{Q-1} x_{\mathrm{U}}^{3}(n-q) x_{\mathrm{L}}^{* 2}(n-q) f_{\mathrm{U} 5}\left(\left|x_{\mathrm{U}}(n-q)\right|^{2},\left|x_{\mathrm{L}}(n-q)\right|^{2}\right),
\end{aligned}
$$

where the gain function $f_{\mathrm{Lm}}\left(\left|x_{\mathrm{L}}(n-q)\right|^{2},\left|x_{\mathrm{U}}(n-q)\right|^{2}\right)$ can be expressed as complex gain of the memory polynomial model (MP), as is similarly done in the conventional one-band [3] and dual-band DPD approaches [5]

$$
\begin{aligned}
& f_{\mathrm{Lm}}\left(\left|x_{\mathrm{L}}(n-q)\right|^{2},\left|x_{\mathrm{U}}(n-q)\right|^{2}\right) \\
& =\sum_{k=0}^{K-m} \sum_{l=0}^{k} \beta_{q, k, l}^{L}\left|x_{\mathrm{L}}(n-q)\right|^{k-l}\left|x_{\mathrm{U}}(n-q)\right|^{l}, m=1,3,5 .
\end{aligned}
$$

Similarly, the gain function $f_{\mathrm{Um}}\left(\left.p_{\mathrm{L}}(n-q)\right|^{2},\left.p_{\mathrm{U}}(n-q)\right|^{2}\right)$ can be expressed as

$$
\begin{aligned}
& f_{\mathrm{Um}}\left(\left|x_{\mathrm{U}}(n-q)\right|^{2},\left|x_{\mathrm{L}}(n-q)\right|^{2}\right) \\
& =\sum_{k=0}^{K-m} \sum_{l=0}^{k} \beta_{q, k, l}^{U}\left|x_{\mathrm{U}}(n-q)\right|^{k-l}\left|x_{\mathrm{L}}(n-q)\right|^{l}, m=1,3,5,
\end{aligned}
$$

where $\beta_{q, k, l}^{L}$ and $\beta_{q, k, l}^{U}$ are the coefficients of the proposed dual-band PA model. Because both bands are uncorrelated [9], the model coefficients can be estimated using the least squares (LS) method. In the case where the receiving bandwidth is wide enough for capturing the inter-band modulation byproducts, all the coefficients can be estimated simultaneously. Otherwise, the measured signals of each band needs to be shifted back to $w_{\mathrm{L}}$ and $w_{\mathrm{U}}$ and the composite signal needs to be reconstructed as is done in conventional dual-band DPD system. To facilitate the matrix inversion process, singular value decomposition (SVD) can be applied to further reduce the coefficient number and mitigate any residual ill-conditioning problem, where principle components of the eigenvalues are considered for matrix inversion solving.

After performing the PA model parameters extraction for both the lower and upper bands, i.e., the $\hat{\beta}_{q, k, l}^{L}$ and $\hat{\beta}_{q, k, l}^{U}$, the PA model estimations for the lower and upper bands, $\hat{y}_{\mathrm{L}}(n)$ and $\hat{y}_{\mathrm{U}}(n)$ can be expressed using (4) and (5). Next using the indirect learning architecture, i.e., by swapping the input and output of the dual-band PA model functions, the DPD models for both bands can also be built using functions (4) and (5), such that the corresponding DPD coefficients can be extracted.

\section{EXPERIMENTAL RESULTS}

The experimental setup used to evaluate the DPD linearization performance is shown in Fig. 2. The PC controls the FPGA board using a USB cable and can download/upload 


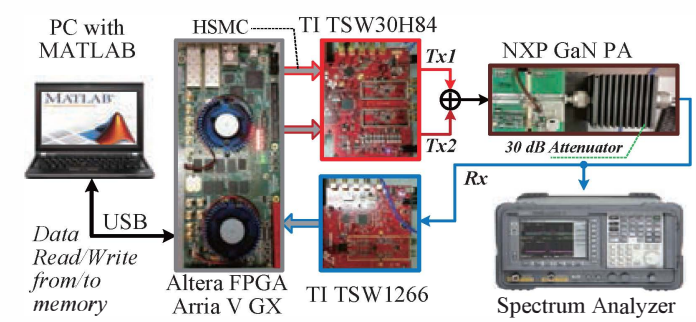

Fig. 2. Experimental setup used to evaluate the behavioral model and DPD.

data from/to the SDRAM memory built on the FPGA board. The baseband signals are generated using Matlab, uploaded to the SDRAM memory, and processed by the FPGA for transmission. Both processed baseband signals are then sent to their respective DAC chain in the TI TSW30HS84 board. The predistorted digital signals are sent to the baseband board, controlled by one Arria V GX-360KLE FPGA, to be upconverted to IF signals centered at $267.2 \mathrm{MHz}$ and $347.2 \mathrm{MHz}$, respectively, which are then processed and modulated by the selected radio frequency carrier. A 10-Watts peak output power amplifier, realized with a NXP Semiconductor GaN HEMT transistor CLF1G0060-10 working at $2.35 \mathrm{GHz}$, is used to amplify the RF signal. In the feedback path, a 30-dB attenuator is used to attenuate the PA output signal, which is subsequently redirected to the receiving board TI TSW1266 to be downconverted, digitalized, and stored in the SDRAM memory. The stored data is then sent back to Matlab to be aligned with the input signals for PA modeling for the DPD coefficient extraction.

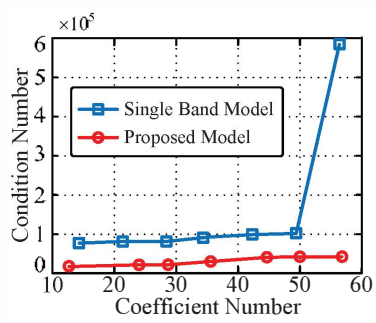

(a)

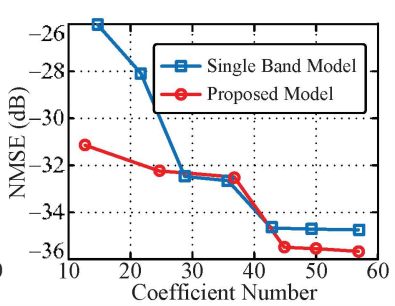

(b)
Fig. 3. (a) Condition numbers versus coefficient number; (b) NMSE performances versus coefficient number.

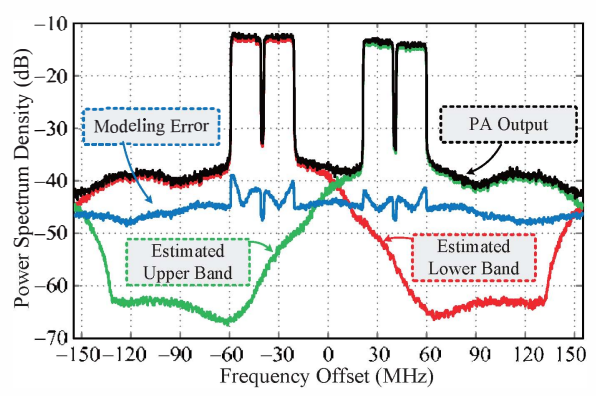

Fig. 4. Dual-band PA modeling performances for lower and upper bands.

The dual-band signal under test is composed of two $40-\mathrm{MHz}$
LTE-advanced signals with carriers separated by $80 \mathrm{MHz}$. In the dual-band PA modeling process, due to the overlap of inband intermodulations and inter-band modulation by-products, the conventional 2D-DPD method will fail to work properly. Therefore, only the conventional one-band MP based DPD is used for comparison. Fig. 3. (a) shows the condition number of the model nonlinear kernel matrix of both methods. Fig. 3 . (b) shows the NMSE performances of the conventional single band method and the proposed one in terms of the number of coefficients. Fig. 4 shows the modeling performances of the proposed dual-band model where the coefficient number is reduced from 186 to 56 while still preserving good modeling performances. The estimated dual-band PA output signals will be used to extract the dual-band DPD coefficients.

To further verify the new model, the conventional 2DDPD method [5] is also considered to linearize the dual-band signal under test, where a digital $80-\mathrm{MHz}$ low-pass filter is used to isolate the lower and upper band PA output from the received signal. Fig. 5 shows the PA output spectra measured by the spectrum analyzer with and without the DPDs. It can be clearly seen that the conventional 2D-DPD fails to work properly due to the overlap of the signals. Also because the wide bandwidth requires higher nonlinear order and memory depth, the conventional single-band method cannot satisfy the spectral mask. On the other hand, the proposed method can effectively model the dual-band PA nonlinearity and memory effect and reduce the spectral regrowth by about $16 \mathrm{~dB}$ for both lower and upper bands, demonstrating by up to $5 \mathrm{~dB}$ improvement over the conventional one-band method.

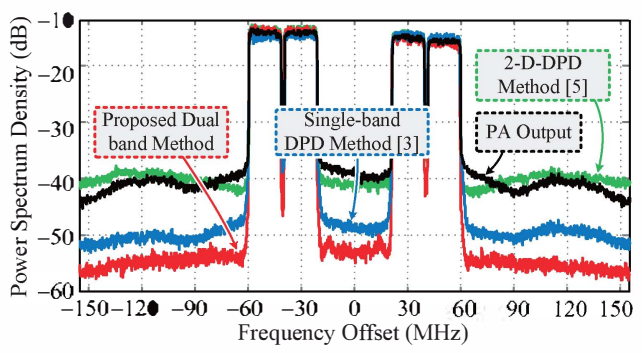

Fig. 5. PA output spectra of different DPD methods.

\section{CONCLUSION}

In this paper, a novel multiband PA model is reported, which can effectively compensate for the nonlinear distortion when the dual-band signals are closely spaced. By including the in-band intermodulation, the cross-modulation, and the inter-band modulation by-products into the PA model, the proposed technique can extract the dual-band PA model from the measured data corrupted by spectral overlap and obtain clean PA outputs for the lower and the upper bands, respectively. Subsequently, by applying the indirect learning method on the estimated dual-band PA outputs and inputs, the dual-band DPD coefficients can be extracted. This method is validated by experimental results which establish that the proposed linearization method can provide improved spectral regrowth cancellation performance up to $5 \mathrm{~dB}$. 


\section{ACKNOWLEDGMENT}

This work was supported in part by the National Science Foundation under Grant ECS 1129013, the National Natural Science Foundation of China under Grants 61471108, 61271164, U1035002/L05, 61001087, 61101034, and 61301154, the National Major Projects under Grants 2014ZX03003001-002, 2012ZX03003010-003, and 2011ZX03001-006-013 and the 863 Project under Grant 2014AA01A704.

\section{REFERENCES}

[1] Z. Shen, A. Papasakellariou, J. Montojo, and F. Xu, "Overview of 3GPP LTE-advanced carrier aggregation for $4 \mathrm{G}$ wireless communications," IEEE Commun. Mag., vol. 50, no. 2, pp. 122-130, Feb. 2012.

[2] R. Liu, D. Schreurs, W. De Raedt, F. Vanaverbeke, and R. Mertens, "Concurrent dual-band power amplifier with different operation modes," IEEE MTT-S Int. Microw. Symp. Dig., Baltimore, MD, USA, Jun. 2011.

[3] F. M. Ghannouchi and O. Hammi, "Behavioral modeling and predistortion," IEEE Microw. Mag., vol. 10, no. 7, pp. 52-64, Dec. 2009.

[4] P. Roblin, S. K. Myoung, D. Chaillot, Y. G. Kim, A. Fathimulla, J. Stahler, and S. Bibyk, "Frequency-selective predistortion linearization of RF power amplifiers," IEEE Trans. Microw. Theory Techn., vol. 56, no. 1, pp. 65-76, Jan. 2008.

[5] S. A. Bassam, M. Helaoui, and F. M. Ghannouchi, "2-D digital predistortion (2-D-DPD) architecture for concurrent dual-band transmitters," IEEE Trans. Microw. Theory Techn., vol. 59, no. 10, pp. 2547-2553, Oct. 2011.

[6] Y. Liu, W. Chen, J. Zhou, B. Zhou, and F. M. Ghannouchi, "Digital predistortion for concurrent dual-Band transmitters using 2-D modified memory polynomials," IEEE Trans. Microw. Theory Techn., vol. 61, no. 1, pp. 281-290, Jan. 2013.

[7] S. A. Bassam, M. Helaoui, and F. M. Ghannouchi, "Channel-selective multi-cell digital predistorter for multicarrier transmitters," IEEE Trans. Commun., vol. 6, no. 8, pp. 2344-2352, Aug. 2012.

[8] P. Roblin, C. Quindroit, N. Naraharisetti, Shahin Gheitanchi, and M. Fitton, "Concurrent linearization," IEEE Microw. Mag., vol. 14, no. 7, pp. 75-91, Nov. 2013.

[9] C. Quindroit, M. Rawat, N. Naraharisetti, P. Roblin, S. Cheitanchi, and D. Chaillot, "Digitally modified filter-less receiver for 2D digital predistortion Of concurrent dual-band power amplifiers," IEEE MTT-S Int. Microw. Symp. Dig., Tampa, FL, USA, Jun. 2014. 